FIT HE - 12-03

KYUSHU-HET 135

SAGA-HE-275

\title{
Holographic cold nuclear matter as dilute instanton gas
}

\author{
Kazuo Ghoroku诖, Kouki Kubo圤, Motoi Tachibana Q 3 , \\ Tomoki Taminato特, and Fumihiko Toyoda 45 \\ ${ }^{\dagger}$ Fukuoka Institute of Technology, Wajiro, Higashi-ku \\ Fukuoka 811-0295, Japan \\ $\ddagger_{D}$ Department of Physics, Kyushu University, Hakozaki, Higashi-ku \\ Fukuoka 812-8581, Japan \\ ${ }^{Q}$ Department of Physics, Saga University, Saga 840-8502, Japan \\ Faculty of Humanity-Oriented Science and Engineering, Kinki University, \\ Iizuka 820-8555, Japan
}

\begin{abstract}
We study cold nuclear matter based on the holographic gauge theory, where baryons are introduced as the instantons in the probe $\mathrm{D} 8 / \overline{\mathrm{D} 8}$ branes according to the SakaiSugimoto model. Within a dilute gas approximation of instantons, we search for the stable states via the variational method and fix the instanton size. We find the first order phase transition from the vacuum to the nuclear matter phase as we increase the chemical potential. At the critical chemical potential, we could see a jump in the baryon density from zero to a finite definite value. While the size of the baryon in the nuclear matter is rather small compared to the nucleus near the transition point, where the charge density is also small, it increases with the baryon density. Those behaviors obtained here are discussed by relating them to the force between baryons.
\end{abstract}

\footnotetext{
${ }^{1}$ gouroku@dontaku.fit.ac.jp

${ }^{2}$ kkubo@higgs . phys . kyushu-u.ac.jp

${ }^{3}$ motoi@cc.saga-u.ac.jp

${ }^{4}$ taminato@higgs.phys.kyushu-u.ac.jp

${ }^{5}$ ftoyoda@fuk.kindai.ac.jp
} 


\section{Introduction}

It is difficult to study dense nuclear matter from the 4D non-perturbative approach like lattice gauge theory due to the sign problem, which appears when we introduce the chemical potential (See for example [1, 2]). Then it is challenging to make clear the properties of the nuclear matter from the viewpoint of holographic gauge theory.

In this approach, baryons can be introduced as solitons on the probe flavor branes [3, 4, 5, 6]. For the simplicity, we consider two-flavor case, $N_{f}=2$. The soliton, which carries unit baryon number, is given as the BPST instanton solution of the $S U\left(N_{f}\right) \mathrm{YM}$ theory in the flat 5D space-time of the probe brane [4]. The instanton configuration has a scale parameter which could be identified with the baryon size. In the case of flat space, the size is free. On the other hand, the geometry of the world-volume of the embedded flavored brane is deformed in the fifth direction. As a result, the size of the instanton is fixed by taking into account of this deformation. Actually, in [4], this size of the baryon is determined by minimizing the action of the probe $\mathrm{D} 8 / \overline{\mathrm{D} 8}$, which is expressed as a sum of the Dirac-Born-Infeld (DBI) and the Chern-Simons (CS) terms. The CS term is also necessary to introduce the baryon chemical potential in this approach with the instantons, because the instantons, i.e. baryons, and the $U(1)$ gauge field dual to the baryon number current are connected via the CS term.

In this context, several approaches to study the nuclear system have been performed by introducing the chemical potential of baryons [7, 8, 9, 10, 11, 12, 13, 14]. In [8], the instantons are, however, used to give a delta-function type source at the bottom of the D8 brane configuration only in the CS term. Furthermore, D4 branes are added to cancel the singularity generated by the delta-function type of source at this point, then the $\mathrm{V}$-shaped D8/ $\overline{\mathrm{D} 8}$-brane configuration is obtained instead of the smooth U-shaped one. As a result, the authors of [8] have observed a gapless transition from the vacuum to the nuclear matter phase at zero temperature. However this point is different from other many kinds of theories (See for example [15]).

On the other hand, the authors of [10] have preserved the flavor gauge fields in the DBI action as well as in the CS term, then they could find the first order phase transition at finite baryon density. However, the configuration used for the flavored gauge fields is obscure, so that it is difficult to see the relation between it and the well known instanton. Furthermore, the profile of D8 brane is not solved in the form of U-shape one, which should depend on the gauge fields configurations in the same DBI action.

Here we introduce the explicit form of instanton solution, which is exact in the flat 4D space (our three-space and fifth one), by keeping its size parameter. Then it has a smooth configuration in the fifth coordinate direction instead of the delta function form. Furthermore, the flavored Yang-Mills fields are retained as instantons in the DBI action of the D8 probe brane in its leading order, namely up to the square of the field strength. The latter point is crucial to find a gap of the baryon density at the transition 
point as in the case of [10]. Differently from [10], in our approach, the flavored YM field is solved by determining the remained size parameter of the instanton. After that, the physical quantities like chemical potential and baryon number density are obtained to search for the phase transition.

As for the profile of $\mathrm{D} 8 / \overline{\mathrm{D} 8}$ brane, it is restricted here to the antipodal solution. Then, we can use a simple D8 brane profile [4], which is obtained without any instantons as an antipodal U-shaped configuration. In general, the lowest energy solution for some finite baryon density $n$ is not equivalent to the simple solution mentioned above, since it is not antipodal. However, the solution is always approximated by the simple one with a negligible correction for any $n$. This implies that the simple solution is useful at any $n$ as the D8/ $\overline{\mathrm{D} 8}$ antipodal configuration in our analysis. This is the reason why we restrict the profile to the antipodal solution. Another reason is for the simplicity of the present analysis.

Through our analysis, we find a first order phase transition, which is expressed in $\bar{n}-\mu$ plane, where $\bar{n}$ and $\mu$ denote the vacuum expectation value of charge density (times some dimensionful constant) and the chemical potential. At the transition point, the baryon density jumps from zero to a finite value, which corresponds to the transition from the vacuum to a nuclear matter phase. By adjusting the parameters, at the transition point, we observe the baryon mass as $\mu_{B} \sim 2.3 \mathrm{GeV}$ which is rather large compared to the realistic nucleon mass. Furthermore at this transition point, the size of the baryon is rather small, but it increases as $\sqrt{\mu}$ at large $\mu$.

The outline of this paper is as follows. In the next section, the dilute instanton gas model is set up. Then, the embedded solution of the D8 profile and other fields are given in the section 3. In the section 4, energy density of the system is examined by using a simple profile solution to find phase transitions. In the final section, summary and discussions are given.

\section{Dilute instanton gas model}

Here baryon is identified with an instanton [4]. Then we approximate multi-baryon system by the dilute gas system of multi-instantons. The system is described by using D8 branes with the following action.

\subsection{DBI action}

For stacked two branes,

$$
S_{D_{p}}=-T_{p} \int d^{p+1} \xi^{a} e^{-\Phi} \operatorname{Str} L
$$


where $p=7(8)$ for type $\operatorname{IIB}(\mathrm{A})$, and Str denotes the symmetric trace of flavor $U(2)$

$$
\begin{aligned}
L & =\sqrt{-\operatorname{det}\left(f_{0}+\vec{f}_{1}\right)}, \\
\left(f_{0}\right)_{a b} & =\left(G_{M N} \partial_{a} X^{M} \partial_{b} X^{N}+B_{a b}+\tilde{F}_{a b}\right) \tau_{0}, \\
B_{a b} & =B_{M N} \partial_{a} X^{M} \partial_{b} X^{N}=-B_{b a} \\
\tilde{F}_{a b} & =2 \pi \alpha^{\prime} F_{a b}, \\
\vec{f}_{1} & =2 \pi \alpha^{\prime} F_{a b}^{i} \tau_{i},
\end{aligned}
$$

where $a, b=0 \sim p, M, N=0 \sim 9$, and $\tau_{0}, \tau_{i}$ are the unit, Pauli's spin matrices. The Str part is expanded as

$$
\begin{aligned}
\operatorname{Str} L & =\operatorname{Str}\left\{\sqrt{-\operatorname{det}\left(f_{0}\right)}\left(1-\frac{1}{4} \operatorname{tr} x^{2}+\frac{1}{8}(\operatorname{tr} x)^{2}+\cdots\right)\right\}, \\
x & =f_{0}^{-1} \vec{f}_{1},
\end{aligned}
$$

where 'tr' denotes the trace of the coordinate index. The linear term vanishes for Str, then it is dropped. The coordinates are set as

$$
\left(\xi^{0}, \xi^{1}, \xi^{2}, \xi^{3}, \xi^{4}, \cdots\right)=\left(x^{0}, x^{1}, x^{2}, x^{3}, z, \cdots\right)
$$

and we make the ansatz

$$
\begin{aligned}
\tilde{F}_{a b} & =2 \partial_{[a} A_{b]}, \quad B_{a b}=0, \\
A_{b} & =A_{b}(z) \delta_{b}^{0}, \\
\left(\overrightarrow{f_{1}}\right)_{i j} & =Q\left(x^{m}-a^{m}, \rho\right) \epsilon_{i j k} \tau^{k}, \\
\left(\overrightarrow{f_{1}}\right)_{i z} & =Q\left(x^{m}-a^{m}, \rho\right) \tau^{i},
\end{aligned}
$$

where $\rho\left(a^{m}\right)$ denotes the instanton size (position), $\epsilon_{123 z}=1, i, j=1,2,3$ and $m=$ $1, \ldots, 4$, where $x^{4}=z$.

Then the above series of $\vec{f}_{1}$, by retaining its lowest order, are obtained as

$$
\begin{aligned}
\operatorname{Str} L & =2 \sqrt{-\operatorname{det}\left(f_{0}\right)}\left(1+\frac{3}{2} Q^{2}\left[\left(\mathcal{G}^{11}\right)^{2}+\mathcal{G}^{11} \mathcal{G}^{z z}\right]\right), \\
\mathcal{G}_{a b} & =G_{M N} \partial_{a} X^{M} \partial_{b} X^{N} .
\end{aligned}
$$

Hereafter we neglect the higher order terms of the non-Abelian gauge fields.

\section{2 $Q$ as a Dilute Gas of Instantons}

In order to see the baryon spectrum, $Q$ is given as an instanton solution in flat $4 \mathrm{D}$ space $\left\{x^{m}\right\}$ [4,

$$
Q=\frac{2 \rho^{2}}{\left(\left(x^{m}-a^{m}\right)^{2}+\rho^{2}\right)^{2}} .
$$


However, it is not a solution of the equation of motion given by the D8 brane action. We use it as a trial function which is supposed to be a solution of the system. This is confirmed in our formulation such that the size parameter $\rho$ of the given instanton configuration is determined to satisfy the variational principle of the energy density up to the leading order of the expansion given in (2.13).

According to the above strategy, we study multi-baryon state by replacing $Q(z)$ by the multi-instanton form with dilute gas approximation. Then we rewrite $Q$ as

$$
Q^{2}=\sum_{i}^{N_{I}} \frac{4 \rho^{4}}{\left(\left(x^{m}-a_{i}^{m}\right)^{2}+\rho^{2}\right)^{4}},
$$

where the overlapping between the instantons are suppressed in obtaining $Q^{2}$. So we find the energy density in the flat $4 \mathrm{D}$ space as a sum of each single instanton,

$$
\begin{aligned}
\int d^{4} \xi^{m} Q^{2} & =2 N_{I} \int_{0}^{\infty} d z \bar{q}(z)^{2} \\
& =\frac{2 \pi^{2}}{3} N_{I}
\end{aligned}
$$

where

$$
\bar{q}^{2}=\frac{\pi^{2} \rho^{4}}{2\left(z^{2}+\rho^{2}\right)^{5 / 2}}
$$

for the case of dilute gas, where interactions between instantons are neglected. Then the result is given by one instanton "mass" times their number $N_{I}$.

As for the size parameter $\rho$, we determine it by minimizing the embedded D 8 brane energy as in [4]. We notice that there is another method giving $Q$ including its $z$ dependence by solving the embedding equations of motion [10]. We, however, solve only for the size parameter here.

\subsection{CS term}

Supposing a reduced 5D action, we consider the following form of CS term for $N_{f}=2$ [4],

$$
S_{C S}=\frac{N_{c}}{24 \pi^{2}} \epsilon^{m_{1} \cdots m_{4}} \int d^{4} x d z \frac{3}{4} A_{0} \operatorname{Tr}\left(F_{m_{1} m_{2}} F_{m_{3} m_{4}}\right) .
$$

For the instanton configuration, we have

$$
S_{C S}=\frac{3 N_{c}}{2 \pi^{2}} \int d^{4} x d z A_{0} \frac{Q^{2}}{\left(2 \pi \alpha^{\prime}\right)^{2}} .
$$

This term is then included in our calculation as the coupling of $A_{0}$ and instantons by supposing $Q=Q(z)$ as in the DBI term. 


\section{Solution of embedded D8/ $\overline{\mathrm{D} 8}$ brane}

Induced metric for D8 brane is given as [3, 4],

$$
\begin{gathered}
d s_{9}^{2}=\frac{\lambda l_{s}^{2}}{3}\left(\frac{4}{9} k^{1 / 2}(z) \eta_{\mu \nu} d x^{\mu} d x^{\nu}+g(\tau) \frac{4}{9} k^{-5 / 6}(z) d z^{2}+k^{1 / 6}(z) d \Omega_{4}^{2}\right), \\
k(z)=1+z^{2}, \quad e^{-\Phi}=\frac{3^{3 / 2} \pi N_{c}}{\lambda^{3 / 2}} k(z)^{-1 / 4}
\end{gathered}
$$

where $k \equiv U^{3}=U_{K K}^{3}+U_{K K} z^{2}$ and we set

$$
U_{K K}=1, \quad R^{3}=\frac{9}{4}=\pi g_{s} l_{s}^{3} N_{c}, \quad \lambda=3 \sqrt{\frac{\pi g_{s} N_{c}}{l_{s}}}, \quad M_{K K}=\frac{3 U_{K K}^{1 / 2}}{2 R^{3 / 2}},
$$

where $l_{s}$ and $g_{s}$ are the string length and the string coupling respectively 1 In this notation, we use $\lambda l_{s}^{2} / 3=3 / 2, \alpha^{\prime}=9 / 2 \lambda$. Moreover, we give

$$
g(\tau)=1+z^{2} k^{1 / 3} \tau^{\prime 2}, \quad \tau^{\prime}=\frac{\partial \tau(z)}{\partial z} .
$$

Note that $\tau$ should have a periodicity $\tau \sim \tau+\delta \tau$ in order to avoid a conical singularity at $z=0$. In the case of (3.1),$\delta \tau$ is denoted as $2 \pi[3$.

Then we have

$$
\begin{aligned}
S_{D 8} & =-T_{p} \int d^{9} \xi^{a} e^{-\Phi} \operatorname{Str} L \\
& =-\frac{N_{c} \lambda^{3}}{3^{9} \pi^{5}} \int d^{4} x d z k^{\frac{1}{12}}(z) \operatorname{Str} \sqrt{-\operatorname{det}\left(g_{M N}^{(5)}+\tilde{F}_{M N}\right)}, \\
g_{M N}^{(5)} d x^{M} d x^{N} & =k^{1 / 2}(z) \eta_{\mu \nu} d x^{\mu} d x^{\nu}+g(\tau) k^{-5 / 6}(z) d z^{2} \\
\tilde{F}_{M N} & \equiv \frac{3}{2}\left(2 \pi \alpha^{\prime}\right) F_{M N}=\frac{27 \pi}{2 \lambda} F_{M N}
\end{aligned}
$$

Using the above formula, we find

$$
S_{D 8}=-2 \kappa \int d^{4} x d z k^{5 / 6}(z) \sqrt{g(\tau) k^{-1 / 3}(z)-\tilde{E}_{z}^{2}}\left(1+\frac{3}{2} \tilde{Q}^{2}\left(k^{-1}+k^{1 / 3} g(\tau)^{-1}\right)\right),
$$

where $\tilde{E}_{z}=\tilde{F}_{z 0}=\partial_{z} \tilde{A}_{0}(z)$ and $\tilde{Q}=\frac{3}{2} Q$. Similarly we obtain

$$
S_{C S}=\frac{2^{2} \lambda^{3} N_{c}}{3^{8} \pi^{5}} \int d^{4} x d z \tilde{A}_{0}(z) \tilde{Q}^{2} .
$$

Hereafter we drop all tildes for simplicity.

\footnotetext{
${ }^{1}$ We notice that $z$ is taken as a world-volume coordinate of the D8 brane, and $\tau$ is set as the external one. Then it is included in the induced metric of D8 brane as a scalar field $\tau(z)$.
} 
The effective action is given as

$$
\begin{aligned}
S & =S_{D 8}+S_{C S}=-2 \kappa V_{3} \int d t d z L_{Q}, \\
L_{Q} & =k^{5 / 6} \sqrt{g(\tau) k^{-1 / 3}-E_{z}^{2}} Q_{1}-6 A_{0} n \bar{q}_{0}^{2}, \\
Q_{1} & =1+\frac{3}{2} n \bar{q}_{0}^{2}\left(k^{-1}+k^{1 / 3} g(\tau)^{-1}\right),
\end{aligned}
$$

where $V_{3}$ is the volume of 3-dimensional space and $n$ is the density of instantons defined by

$$
n=\frac{N_{I}}{V_{3}} .
$$

Note here that we replaced the previous instanton configuration $\bar{q}^{2}$ to the following new form

$$
\bar{q}_{0}^{2}=\frac{9}{8} \frac{\pi^{2} \rho^{4}}{\left(\left(z-z_{0}\right)^{2}+\rho^{2}\right)^{5 / 2}},
$$

which is reduced to $\bar{q}^{2}$ for $z_{0}=0$. In any case, we take the position of the inserted instantons at the bottom of the $\mathrm{D} 8$ brane since they are pulled to the horizon by the gravitational force. In other words, we put the instantons at their stable position. On the other hand, they are distributed at random points in the three space of our Minkowski space-time.

\subsection{Equations of motion for the system $S=S_{D 8}+S_{C S}$}

We suppose the form (3.14) for the instanton contribution, where the parameter $\rho$ is remained to be determined. It is given here by minimizing the action.

The equations of motion for $A_{0}(z)$ and $\tau(z)$ are given as

$$
\begin{gathered}
\partial_{z}\left(k^{5 / 6} \frac{E_{z}}{\sqrt{g(\tau) k^{-1 / 3}-E_{z}^{2}}} Q_{1}\right)=6 n \bar{q}_{0}^{2} \\
\partial_{z}\left(k^{5 / 6} \frac{z^{2} \tau^{\prime}}{\sqrt{g(\tau) k^{-1 / 3}-E_{z}^{2}}} Q_{1}\right)-3 n \partial_{z}\left(k^{3 / 2} \sqrt{g(\tau) k^{-1 / 3}-E_{z}^{2}} \frac{\bar{q}_{0}^{2} z^{2} \tau^{\prime}}{g^{2}(\tau)}\right)=0 .
\end{gathered}
$$

At first, we solve for $A_{0}(z)$, whose equation of motion is given as

$$
\begin{gathered}
k^{5 / 6} \frac{E_{z}}{\sqrt{g(\tau) k^{-1 / 3}-E_{z}^{2}}}\left(1+\frac{3}{2} n \bar{q}^{2}\left(k^{-1}+k^{1 / 3} / g(\tau)\right)\right)=d, \\
d=6 n \int_{z_{0}}^{z} d z \bar{q}_{0}^{2}+C=\frac{9}{4} n \pi^{2}\left(z-z_{0}\right) \frac{2\left(z-z_{0}\right)^{2}+3 \rho^{2}}{\left(\left(z-z_{0}\right)^{2}+\rho^{2}\right)^{3 / 2}}+C .
\end{gathered}
$$


Here we take the integration constant $C$ as zero since the displacement would vanish when instantons disappears ( or $n=0$ ). Then we have

$$
E_{z}=\frac{g^{1 / 2} k^{-1 / 6}}{\sqrt{1+f^{2}}}, \quad f=\frac{k^{5 / 6}}{d} Q_{1} .
$$

In the next, by substituting the above $E_{z}$ into (3.16), we find

$$
\frac{z^{2} k \tau^{\prime}}{g^{1 / 2}(\tau)}\left\{\frac{\sqrt{1+f^{2}}}{f} Q_{1}-\frac{f}{\sqrt{1+f^{2}}} \frac{3 n k^{1 / 3} \bar{q}_{0}^{2}}{g(\tau)}\right\}=c_{0},
$$

where $c_{0}$ denotes an integration constant, which is related to the bottom coordinate $z_{0}$. The solution $\tau(z)$ has in general has a U-shape configuration in the $\tau-z$ plane. And, we find $\tau^{\prime}\left(z_{0}\right)=\infty$ at its bottom point, then the following relation is obtained from Eq. (3.20)

$$
z_{0} Q_{1}\left(z_{0}\right) k^{3 / 4}\left(z_{0}\right)=c_{0}
$$

\subsection{Simple solution in the antipodal case}

Here we consider the antipodal solution which satisfies $\tau(\infty)=\pi / 2$ (or $-\pi / 2$ ). The explicit and exact (U-shaped) form of this solution is given as the solution of Eq.(3.20) at $n=0$ and in the limit of $z_{0} \rightarrow 0$. It is approximately given as

$$
\tau^{\prime}(z)=0 \quad \text { for } \quad z>z_{0}
$$

In general, the configuration of the solution $\tau(z)$ depends on the instanton density $n$, and $\tau(\infty)$ increases with $n$ when $z_{0}$ is kept small. Therefore we consider here a new type of solution in order to obtain the antipodal solution in the limit of $z_{0} \rightarrow 0$. In this case, all such solutions are approximated here by a simple solution of Eq.(3.20). 2

On the other hand, we can consider other antipodal solutions that satisfy $\tau(\infty)=$ $\pi / 2$ at finite $z_{0}$ and $n$. However, it is confirmed by numerical analyses that the energy of the solution at finite $z_{0}$ is always larger than the solution (3.22) in the same $n$. Therefore, we adopt here the solution (3.22).

Then $E_{z}$ and the chemical potential are obtained by noticing $g(\tau)=1$ as

$$
\begin{aligned}
E_{z} & =\frac{k^{-1 / 6} d}{\sqrt{Q_{0}^{2} k^{5 / 3}+d^{2}}}, \\
Q_{0} & =1+\frac{3}{2} n \bar{q}_{0}^{2}\left(k^{-1}+k^{1 / 3}\right), \\
\mu & =\mu_{c}+\int_{0}^{\infty} d z E_{z} .
\end{aligned}
$$

\footnotetext{
${ }^{2}$ The details of the solutions and justification of this approximation are given in the Appendix A
} 
Here the quark chemical potential $3 \mu$ and $\mu_{c}$ are defined as

$$
\mu=A_{0}(\infty), \quad \mu_{c}=A_{0}(0) .
$$

In this case, $E_{z}$ is expanded at large $z$ as follows

$$
E_{z}=\frac{9 n \pi^{2}}{2}\left(\frac{1}{z^{2}}-\frac{1}{z^{4}}+\cdots\right),
$$

Here we notice the coefficients of the leading and sub-leading orders are independent of $\rho$. Then we obtain

$$
A_{0}=\mu-\frac{9 n \pi^{2}}{2} \frac{1}{z}+\cdots .
$$

The charge density is expected to correspond to the coefficient of the sub-leading term as

$$
c\left\langle\Psi^{\dagger} \Psi\right\rangle=\bar{n}=\frac{9 n \pi^{2}}{2},
$$

where $c$ is a dimensionful coefficient obtained by returning dimensionless variables to dimensionful ones.

In the small $z$ region, $E_{z}$ is expanded as

$$
E_{z}=\frac{9 n \pi^{2}}{2}\left(a_{1} z+a_{2} z^{3}+\cdots\right),
$$

where $a_{1,2}$ are the functions of $\rho$. Then we find the asymptotic solution of $A_{0}$,

$$
A_{0}=\mu_{c}+\frac{9 n \pi^{2} a_{1}}{4} z^{2}+\cdots .
$$

where $\mu_{c}$ is undetermined.

\section{Energy density and phase transition}

The Chern-Simons term is rewritten as

$$
\begin{aligned}
-6 n \int_{0}^{\infty} d z A_{0} \bar{q}_{0}^{2} & =-6 n \int_{0}^{\infty} d z A_{0}\left(\partial_{z} q_{b}\right) \\
& =6 n\left(\int_{0}^{\infty} d z E_{z} q_{b}-\frac{3 \pi^{2}}{4} \mu\right) \\
& =6 n\left(\int_{0}^{\infty} d z E_{z}\left(q_{b}-\frac{3 \pi^{2}}{4}\right)-\frac{3 \pi^{2}}{4} \mu_{c}\right),
\end{aligned}
$$

\footnotetext{
${ }^{3}$ We notice here that the chemical potential given above expresses the one of the quark rather than that of the baryon.
} 
where

$$
q_{b}=\frac{3 \pi^{2}}{8} \frac{2\left(z-z_{0}\right)^{3}+3\left(z-z_{0}\right) \rho^{2}}{\left(\left(z-z_{0}\right)^{2}+\rho^{2}\right)^{3 / 2}}
$$

and we used $q_{b}(\infty)=\pi^{2} / 3, q_{b}\left(z_{0}\right)=0$ and Eq.(3.25). We notice about the sign of the $S_{C S}$ term. When it is taken as minus as given above, and then we use the selfdual instanton solution. In this case we find positive chemical potential as shown in Eq.(3.25). On the other hand, for opposite sign and self-dual solution, we obtain opposite sign chemical potential

$$
\mu=\mu_{c}-\int_{0}^{\infty} d z E_{z}
$$

and the same sign energy density except for the chemical potential part,

$$
+6 n \int_{0}^{\infty} d z A_{0} \bar{q}_{0}^{2}=6 n\left(\int_{0}^{\infty} d z E_{z}\left(q_{b}-\frac{\pi^{2}}{3}\right)+\frac{\pi^{2}}{3} \mu_{c}\right) .
$$

When we use anti-self dual solution, then the same expression is obtained for the opposite sign. So we must use the sign in our case, we should use the self-dual solution (baryon). When we apply the same analysis for the anti-baryon, we must change the sign of $S_{C S}$ and use the anti-self dual solution. Then we find the same result in both cases.

By using the above solution for $A_{0}$, we define the energy density as

$$
E(n)=V_{3}\left(\int d z L_{Q}-\bar{n} \mu_{c}\right)
$$

where $L_{Q}$ is given as

$$
\begin{aligned}
L_{Q} & =L_{D B I}+L_{C S}, \\
L_{D B I} & =\frac{k^{2 / 3} Q_{0}^{2}}{\sqrt{Q_{0}^{2}+k^{-5 / 3} d^{2}}}, \\
L_{C S} & =6 n d \frac{q_{b}-\frac{3 \pi^{2}}{4}}{k \sqrt{Q_{0}^{2}+k^{-5 / 3} d^{2}}} .
\end{aligned}
$$

Then, we compare the energy density $E(n)$ with $E(0)$ to find the critical value of chemical potential, $\mu_{c r}$. Above which we will find $E(n)<E(0)$ then the baryon phase is realized there.

The integration of the Lagrangian $L_{D B I}$ for finite $n$ diverges, so we need a subtraction, which can be chosen as the energy density of the vacuum with $n=0$. $E(0)$ is given as

$$
E(0)=V_{3} \int d z k^{2 / 3}
$$


Then we can perform the calculation of $E(n)-E(0)$ by using $\tilde{L}_{D B I}$,

$$
\tilde{L}_{D B I}=L_{D B I}-k^{2 / 3} .
$$

We can thus estimate the energy difference for a given density $n$ by normalizing as

$$
\mathcal{E}=\int_{0}^{\infty} d z\left[\tilde{L}_{D B I}+L_{C S}\right]-\bar{n} \mu_{c} .
$$

In estimating this energy density, we set $\mu_{c}=A_{0}(0)=0$ since $A_{0}(z)$ must be a two valued function which is symmetric at $z=0$. Performing the integration over $z$ for a fixed $n$, we obtain $\mathcal{E}(\rho)$ as a function of $\rho$.

In the estimation of $\mathcal{E}(\rho)$, we must be careful about the value of $\mathcal{E}(0)$. The value is sensitive to the order of taking the limit of $z \rightarrow z_{0}$ and $\rho \rightarrow 0$. Our estimation is performed by the integration with respect to $z$ from $z_{0}$ to $z=\infty$, then the limit of $\rho \rightarrow 0$ is examined.

\subsection{Phase Transition}

To estimate physical quantity, we must determine the size parameter $\rho$ by minimizing the energy density $\mathcal{E}(\rho)$. This is performed by estimating $\mathcal{E}(\rho)$ for each fixed $\mu$ not for fixed $n$ since the parameter which characterizes the boundary theory is not $n$ but the chemical potential $\mu$. In our formalism, however, the energy density is given as a function of $n$ and $\rho$ from the Eq. (4.10). At the same time, $\mu$ is also obtained as a function of $n$ and $\rho$ from Eq. (3.25). Then $\mathcal{E}(\rho, \mu)$ for a fixed $\mu$ is estimated by using both calculations of $\mathcal{E}(\rho, n)$ and $\mu(\rho, n)$ although it is a little complicated.
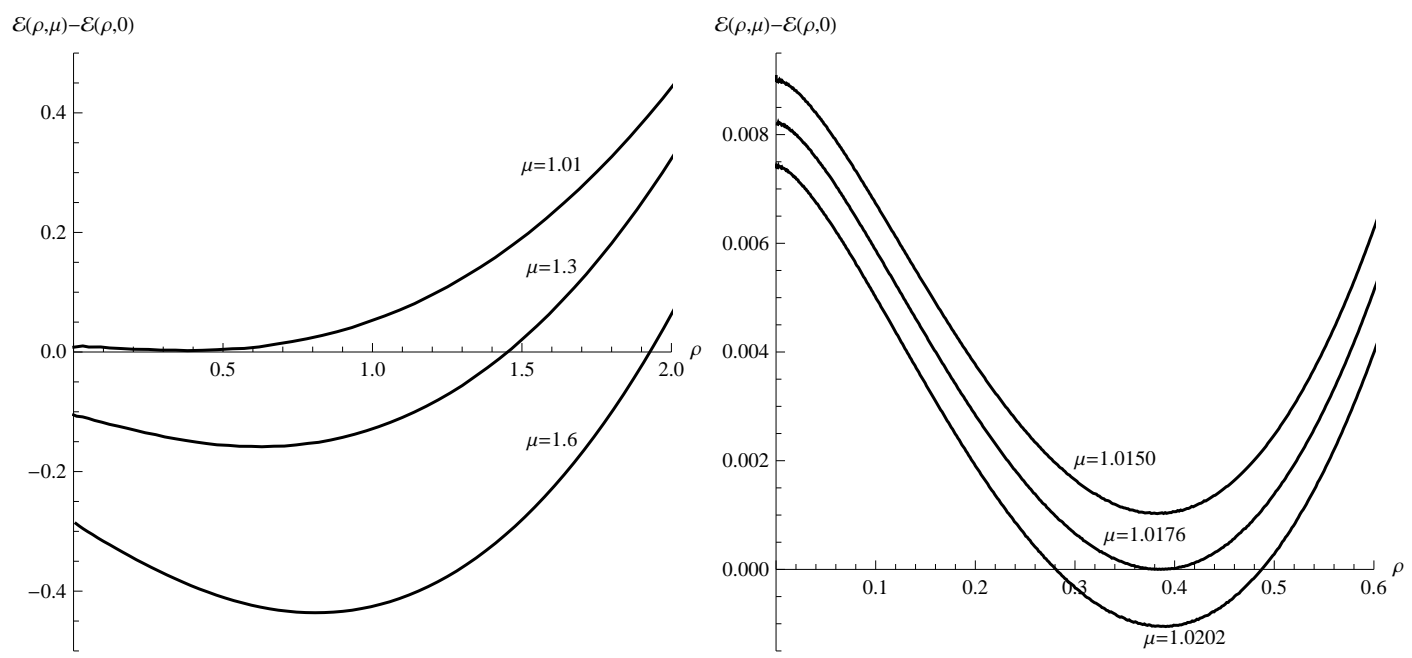

Fig. 1: $\mathcal{E}(\mu)-\mathcal{E}(0)$ versus instanton size $\rho$ near phase transition point $\mu_{c r} \sim 1$. 
Noticing the property of $\mathcal{E}(\rho)$ mentioned above, we estimated $\mathcal{E}(\rho)$ for a fixed $\mu$. Then, fix the the value of $\rho$ as $\rho_{m}$ at the minimum of energy density, namely as $\mathcal{E}_{\min }=\mathcal{E}\left(\rho_{m}\right)$ for the given $\mu$. After that, we determine $\bar{n}$. The resultant curves of $\mathcal{E}(\rho)$ are shown in the Fig. 1 near the phase transition point. The phase transition is observed at $\mu_{c r}=1.0176$, where $\mathcal{E}\left(\rho_{m}\right)$ changes its sign from positive to negative. This implies the transition from the vacuum to the nuclear matter phase at a finite charge density and finite chemical potential, $(\mu, \bar{n})=(1.01,0.85)$. This transition is also seen more clearly from the Fig. 2 represented in the plane of $\mu-\bar{n}$.

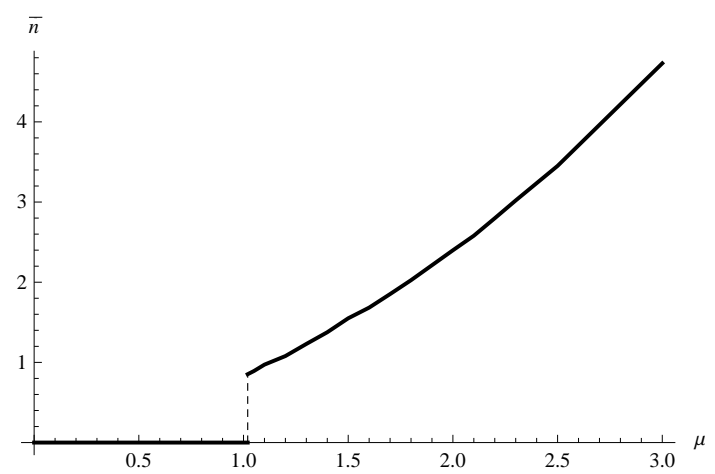

Fig. 2: The diagram of $\left(\mu, \bar{n}=\frac{9 \pi^{2}}{2} n\right)$ for the simple solution. The first order phase transitions, from vacuum to the nuclear matter phase at $\mu_{c r}=1.0176$, is seen.

The reason why $\bar{n}$ is finite at the transition point is reduced to the preserved flavored gauge fields in the DBI action, this is reflected to the factor $Q_{0}>1$ given in (3.24). This point is also mentioned in the introduction. In our model, this is explicitly understood as follows. When we set $Q_{0}=1$, the integrand in (4.10) is expanded near $n=0$ as,

$$
\tilde{L}_{D B I}+L_{C S}=6 n d \frac{q_{b}-3 \pi^{3} / 4}{k}-\frac{1}{2 k} d^{2}+\cdots,
$$

where $\cdots$ denotes the higher order terms of $n$, and notice $d$ is the order of $n$. It is easy to see that the first term of the right hand side of (4.11) is non-positive since

$$
q_{b}-\frac{3 \pi^{3}}{4} \leq 0 .
$$

Here the equality is obtained for $\rho=0$. Then the energy density is always negative for $n>0$ in this case, namely for $Q_{0}=1$. This implies the transition occurs at $n=0$ when we neglect flavored gauge fields in the DBI action. So we can say that the interaction among the baryons as dilute gas is included in the model through the preservation of the flavor gauge field configuration in the probe brane. In spite of the dilute gas approximation adopted, the attractive force to form the nuclear matter phase would be reduced to the curved space in the fifth coordinate direction, namely the interaction reduced to the vacuum of the Yang-Mills theory. Although the details of the dynamics 
should be examined more, the importance of the flavored gauge fields in DBI is clearly understood from our model setting.

\section{Size of baryon}

As for the size of the baryon, which is identified with $\rho_{m}$, its $\mu$ dependence is shown in the left of the Fig. 3. From this numerical results, we can see that $\rho_{m}$ grows with $\mu$ monotonically, and it is fitted by the curve

$$
\rho_{m} \simeq \sqrt{\mu-0.78}-0.10
$$

This implies $\rho_{m}$ increases with the increasing baryon density since $\mu$ grows with $n$. This behavior could be naturally understood by considering the attractive force between the baryons to form the stable nuclear matter. This force becomes large with increasing $n$ since the number of the nearest baryons of one baryon grows. As a result, the attractive force between them increases, and then the size of the baryon is stretched to larger size.

However, at some critical density, baryons begin to overlap each other, then it would become difficult to discriminate each baryon spatially. In this case, we will find a new nuclear phase, but our model cannot be applied to this density since the dilute gas approximation is no longer useful in this region. In the right of the Fig. 3, this point is seen by considering a cube surrounding instanton in three dimensional space which the length is equal to $2 \rho$. We can see from this figure that the dilute gas approximation is not valid any more for $\mu>\mu_{\text {limit }}$. We notice here the following point related to the parameter $\lambda$. Since the critical value of $\mu$ depends on $\lambda$ when we adjust the parameters, we find that the point $\mu_{\text {limit }}$ increases as $\lambda$ deceases. In the case of $\lambda>155$, our model would be no longer valid since $\mu_{\text {limit }}$ become smaller than $\mu_{\mathrm{cr}}$.

\section{Numerical estimation of the size}

In the above analysis, the physical quantities are set to be dimensionless. In this setting, we get

$$
\mu_{c r} \simeq 1.01, \quad n_{c r} \simeq 0.019\left(\bar{n}_{c r} \simeq 0.85\right), \quad \rho_{m} \simeq 0.38
$$

at the critical point. So we must see their dimensionful values to compare them with the realistic physical values, $\left(\mu_{B}=N_{c} \mu, n_{B}, \rho_{B}\right)$.

In our notation, furthermore, we have set as 4

$$
\frac{1}{\sqrt{2 N_{f}}} \frac{27 \pi}{2 \lambda} A_{0} \rightarrow A_{0}, \quad\left(2 \pi \alpha^{\prime}\right)^{2} n=\left(\frac{9 \pi}{\lambda}\right)^{2} n \rightarrow n
$$

\footnotetext{
${ }^{4}$ In reality, we dropped the factor $2 \pi \alpha^{\prime}$ in 2.15). However this factor can be absorbed into $n$ because they always arise together. So we can recover this factor by interpreting $n$ as $\left(2 \pi \alpha^{\prime}\right)^{2} n$.
} 

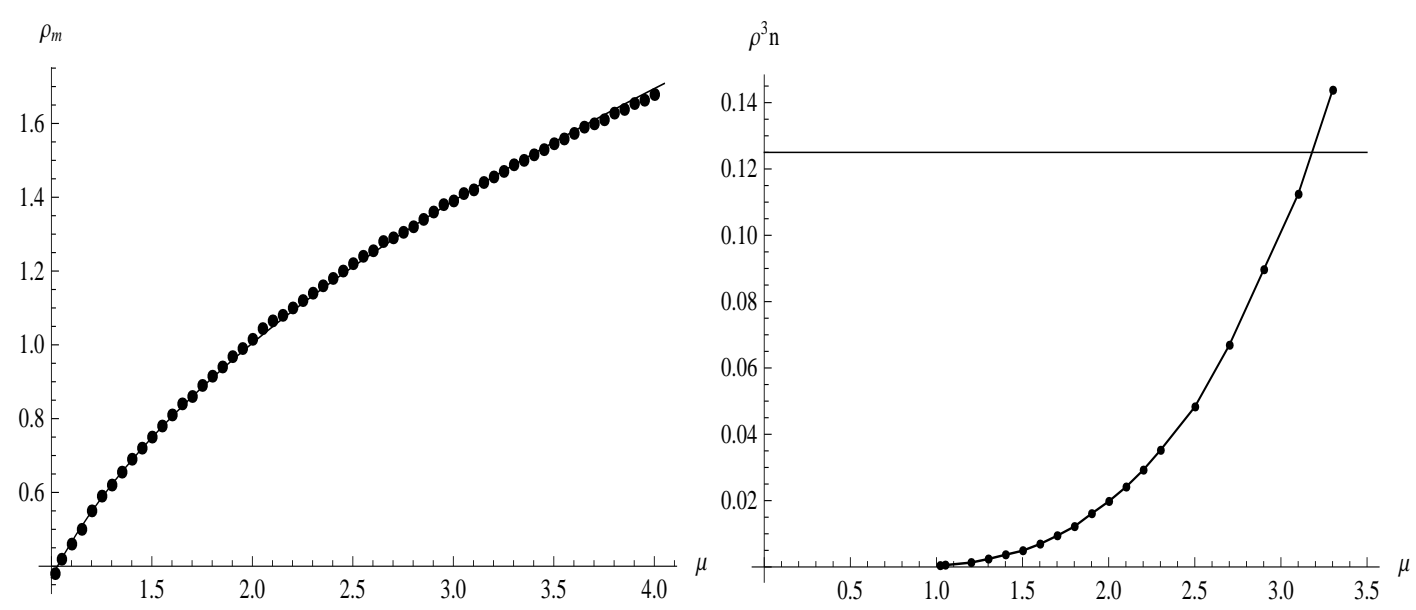

Fig. 3: (Left) The Plot of $\left(\mu, \rho_{m}\right)$. The solid line is given as $\rho_{m}=\sqrt{\mu-0.78}-0.10$. (Right) The relation $\mu-\rho^{3} n$ for $\lambda=16.6$. The horizontal line at $\rho^{3} n=1 / 8$ corresponds to a rough accommodation limit of the dilute gas approximation. The intersection point at about $\mu \sim 3.1$ denotes the useful limit of the model.

Therefore when we compare $\mu_{c r}, n_{c r}$, and $\rho_{m}$ given above with physical quantities of baryon, their scales are given by $M_{K K}$ and $\lambda$ from the following relations as

$$
\begin{aligned}
\frac{\mu_{B}}{M_{K K}} & =\sqrt{2 N_{f}} N_{c} \mu_{c r} \frac{2 \lambda}{27 \pi} \simeq 6 \times 1.01 \times \frac{2 \lambda}{27 \pi}, \\
\frac{n_{B}}{M_{K K}^{3}} & =n_{c r} \frac{1}{\left(2 \pi \alpha^{\prime}\right)^{2}} \simeq 0.019 \times \frac{1}{\left(2 \pi \alpha^{\prime}\right)^{2}}=0.019\left(\frac{\lambda}{9 \pi}\right)^{2}, \\
\rho_{B} M_{K K} & =\rho_{m} \simeq 0.38 .
\end{aligned}
$$

Here we used $N_{f}=2$ and $N_{c}=3$.

In the Sakai-Sugimoto model[3, 5], these parameters are chosen as $M_{K K} \simeq 0.949$ $\mathrm{GeV}$ and $\lambda=16.6$ to fit the values of $\rho$ meson mass and the pion decay constant in the vacuum. Then these parameters lead to

$$
\mu_{B} \simeq 2.3 \mathrm{GeV}, \quad n_{B} \simeq 0.70 \mathrm{fm}^{-3}, \quad \rho_{B} \simeq 0.079 \mathrm{fm}
$$

This value of $n_{B}$ is close to $4 n_{0}$ where $n_{0}=0.16 \mathrm{fm}^{-3}$ is the density of the normal nuclear matter. It is interesting that $4 n_{0}$ corresponds to the density of the inner $\operatorname{core}\left(n>2 n_{0}\right)$ of a neutron star [16].

\section{$5 \quad$ Summary and Discussions}

We have studied cold nuclear matter using the Sakai-Sugimoto model, where the baryon is introduced as a soliton on the probe flavor branes. The soliton, which carries unit 
baryon number, is given by the BPST instanton solution in the probe D8 branes. Multibaryon system is approximated by the dilute gas of instantons in the brane action. The profile of the D8 brane depends on the configuration of the instanton and its density. So it is rather complicated to solve them from the given D8 brane action.

The instanton configuration used here has the same form as the one given in the flat $4 \mathrm{D}$ space, but it is not the solution in the deformed bulk background. So we recovered this point by adjusting the size parameter of the instantons, which is identified with the baryon size. As for the D8 brane profile, we solved it numerically and found that the lowest energy configuration is well approximated by a simple solution, which is obtained without instantons for the antipodal configuration. Through our analysis, we have restricted to this configuration as a boundary condition of the D8 brane which fixes the dual theory.

In our model, we could find the first order phase transition from vacuum to nuclear matter accompanied by a certain jump in the baryon density. This jump could be interpreted as the effect of the attractive force acting among the baryons since a finite density of the instanton gas in the DBI action is needed to form a baryonic system. Actually, we cannot observe the jump in the models which lack such a term in the DBI action. While the density is rather large compared to that of the nucleus, our numerical results obtained near the critical point seems to be reasonable. As a bonus, we could find the size of baryon for each $\mu$. It is rather smaller than the nucleon size just after the nuclear matter is made, but it increases with the density or chemical potential. This observation would be reduced to the increasing attractive force among them.

We should notice on the freedom of parameter choice in determining the physical quantities. For example, the numerical values of $n_{B}$ and $\rho_{B}$ obtained here depend on our parameter choice. If we set $M_{K K} \simeq 0.496 \mathrm{GeV}$ and $\lambda=61.7$ [13], then we get

$$
\mu_{B} \simeq 4.4 \mathrm{GeV}, \quad n_{B} \simeq 1.4 \mathrm{fm}^{-3}, \quad \rho_{B} \simeq 0.15 \mathrm{fm} .
$$

This value of $n_{B}$ is much larger than the ordinary nuclear matter. In this case, we obtain $8 \rho_{B}^{3} n_{B}=0.058$, so the nucleons don't still overlap each other in spite of the high density. Then it is still useful to apply the dilute gas approximation in this parameter choice. Therefore it would be possible to extend our analysis given here to high density nuclear matter like neutron stars. Another important extension is to the finite temperature YM theory by retaining the chemical potential. Then we could see richer phases of nuclear matter. This point will be visited in the near future.

\section{Acknowledgements}

We would like to thank Tetsuo Hatsuda, Shin Nakamura, Kanabu Nawa, Shigeki Sugimoto and Koichi Yazaki for fruitful comments and discussions. M.T. also would like to 
thank the hospitality of Quantum Hadron Physics Laboratory at RIKEN. The work of T.T. is supported by MEXT/JSPS, Grant-in-Aid for JSPS Fellows No. 24.3193. The work of M.T. is supported in part by the JSPS Grant-in-Aid for Scientific Research, Grant No. 24540280.

\section{Appendix}

\section{A The properties of the solution $\tau(z)$}

Here we discuss the solutions $\tau(z)$ and show that the antipodal solutions used here are well approximated by the simple solution (3.22).

\section{A.1 Simple solution; antipodal solution for $n=0$;}

The simple solution is obtained from (3.20) at $n=0$ in the form,

$$
\tau_{(0)}(z)=\int_{z_{0}}^{z} d z \frac{z_{0} k_{0}^{5 / 6}}{z k^{1 / 6} \sqrt{z^{2} k^{5 / 3}-z_{0}^{2} k_{0}^{5 / 3}}}
$$

where $k_{0}=1+z_{0}^{2}$ and $z_{0}^{2} k_{0}^{5 / 3}=c_{0}$. This solution has an U-shape form as shown in the Fig. 6, for example see the one of $z_{0}=10^{-8}$. In the limit of $z_{0} \rightarrow 0$, we find $\tau(\infty)=\pi / 2$ and that the solution is well approximated by (3.22). Since the energetically favourable solution is obtained at very small $z_{0}$ as shown below, then we find that the solutions used in our analysis are approximated by the above simple solution which has originally been given in [3]. In the following, we consider two possible antipodal solutions for finite $n$.

\section{A.2 U-shaped antipodal solutions for finite $n$}

First of all, we study the solutions for finite $n$ by fixing the value of $\tau(\infty)$ at $\pi / 2$. Namely, we examine the variation of the antipodal solutions when the value of $n$ is increased. The numerical results are shown in the Fig. 4. The figure shows that the bottom point $z_{0}$ of the solution increases with $n$ for the boundary condition of

fixed $\tau(\infty)$ (and $\rho$ ). Then we can use these solutions in our analysis. However we must compare solutions when there are two or more solutions with the same boundary 


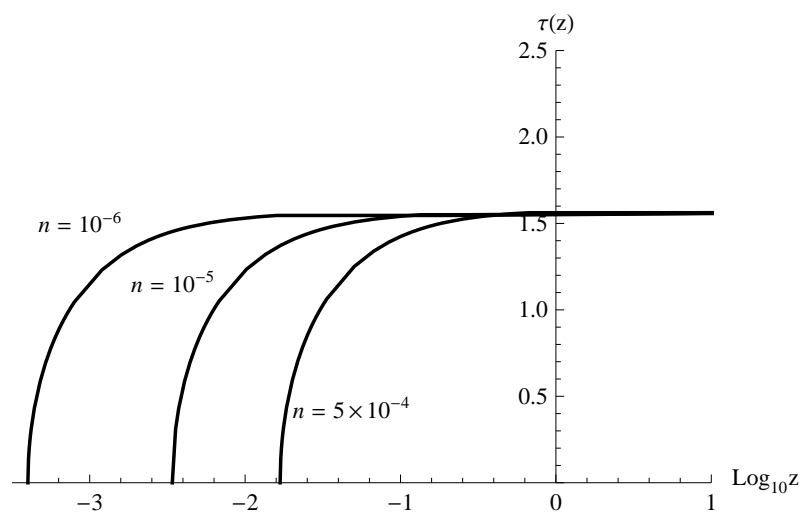

Fig. 4: Typical examples of the U-shaped solutions of $\tau(\infty)=\pi / 2$ for $n=10^{-6}, n=10^{-5}$ and $n=5 \times 10^{-5}$.

condition at $z=\infty$. Then we should adopt the one of the lowest energy density. As shown below, the antipodal solutions given above are abandoned in our analysis since their energy densities are larger than the one of the $\mathrm{V}$-shaped one proposed below.

We show here that the increasing of $z_{0}$ enlarges the energy density of the U-shaped solutions. An example of this behavior is shown in the Fig. 5 for $n=10^{-5}$ and $\rho=0.05$, where we find an antipodal solution at about $z_{0} \simeq 10^{-2.5}$. In the right of Fig. 5 , we show
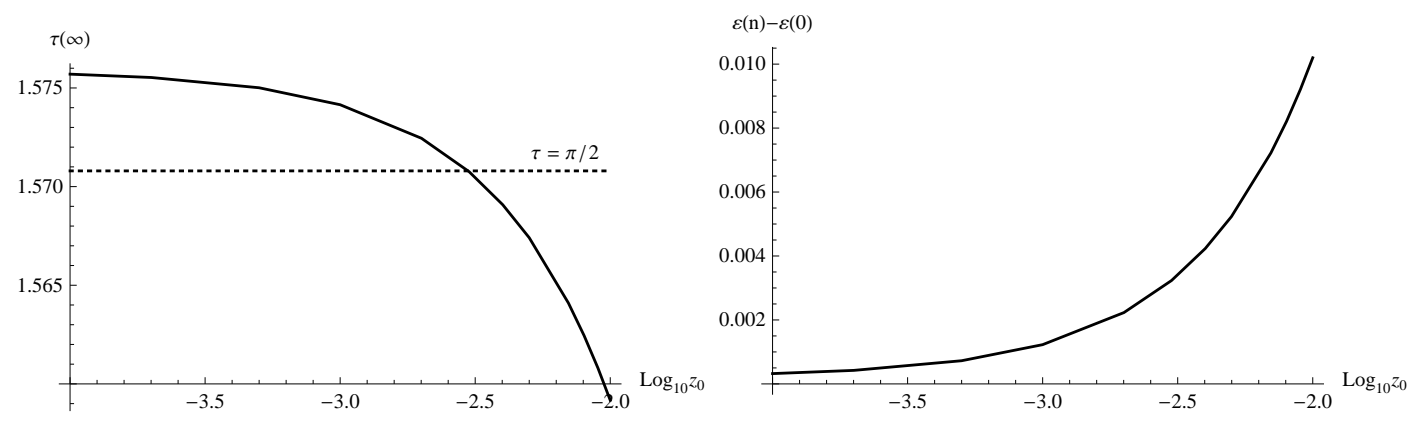

Fig. 5: (Left) $z_{0}$ dependence of $\tau(\infty)$. (Right) $z_{0}$ dependence of $\mathcal{E}(n)-\mathcal{E}(0)$. We take the parameters as $n=10^{-5}$ and $\rho=0.05$ in both figures.

the $z_{0}$ dependence of the energy density $\mathcal{E}(n)-\mathcal{E}(0)$ for the corresponding solutions of the left. It increases with $z_{0} 5$ This result implies that the U-shaped solution with finite $z_{0}$ would be replaced by smaller energy antipodal solution with $z_{0} \rightarrow 0$ if it existed.

\footnotetext{
${ }^{5}$ We give the definition of $\mathcal{E}$ in (4.10). In the present case, we discuss the $n$-dependence of $\mathcal{E}$ and we fix other parameters such as $\rho$. We use $\mathcal{E}(0)$ as a regulator of the energy.
} 


\section{A.3 Finite $n$ solutions in the limit of $z_{0} \rightarrow 0$}

In order to find an antipodal solution with $z_{0} \rightarrow 0$, we study the solution with $z_{0} \rightarrow 0$ for finite $n$. This is equivalent to solve the equation with the boundary condition of $z_{0} \rightarrow 0$. In this case, we find that $\tau(\infty)$ increases with $n$ as shown below.

\section{$n$ dependence of $\tau(\infty)$ for fixed $z_{0}$}

In general, the solution of Eq.(3.20) depends on the instanton density $n$. We can see it by numerical calculation and also by solving the equation expanded perturbatively by the power series of $n$. Especially, we show that $\tau(\infty)$ in the limit $z_{0} \rightarrow 0$ grows with increasing $n$.

First, we expand $\tau^{\prime}$ and $E_{z}$ as follows

$$
\begin{aligned}
E_{z} & =E_{z}^{(0)}+E_{z}^{(1)} n+E_{z}^{(2)} n^{2}+\cdots \\
\tau^{\prime} & =\tau^{\prime(0)}+\tau^{\prime(1)} n+\tau^{\prime(2)} n^{2}+\cdots
\end{aligned}
$$

Then, equations of motion of zeroth order of $n$ are given by setting $n=0$ in the equations (3.15), (3.16). Imposing boundary conditions as $E_{z}^{(0)}\left(z_{0}\right)=0$ and $\tau^{\prime(0)}\left(z_{0}\right)=$ $\infty$, we can solve these equations exactly as follows,

$$
\begin{aligned}
E_{z}^{(0)} & =0 \\
\tau^{\prime(0)} & =\frac{z_{0} k_{0}^{5 / 6}}{z k^{1 / 6} \sqrt{z^{2} k^{5 / 3}-z_{0}^{2} k_{0}^{5 / 3}}}
\end{aligned}
$$

where $k_{0} \equiv k\left(z=z_{0}\right)$ for simplicity. Then, we get $\int_{0}^{\infty} \tau^{\prime(0)} d z=\pi / 2$. The solution $\tau^{(0)}$ at $n=0$ is the exact $\mathrm{U}$-shaped solution, which is the same one given above.

Secondly, we obtain the next order of equations of motion as follows

$$
\begin{gathered}
\partial_{z}\left(k^{5 / 6} \frac{E_{z}^{(1)}}{\sqrt{k^{-1 / 3}+z^{2} \tau^{(0) 2}}}\right)=6 \bar{q}_{0}^{2}, \\
\partial_{z}\left\{\frac{\left(z^{2} k^{5 / 3}-z_{0}^{2} k_{0}^{5 / 3}\right)^{3 / 2}}{z k^{3 / 2}} \tau^{\prime(1)}+\frac{3}{2} z_{0} k_{0}^{5 / 6} \bar{q}_{0}^{2} k^{-1}\left(1-\frac{z^{2} k^{5 / 3}-z_{0}^{2} k_{0}^{5 / 3}}{z^{2} k^{1 / 3}}\right)\right\}=0 .
\end{gathered}
$$

We should notice that $\tau^{\prime(1)}$ in Eq. A.7) is independent of $E_{z}^{(1)}$ because of $E_{z}^{(0)}=0$. Therefore we can obtain $\tau^{\prime(1)}$ from Eq. (A.7) as

$$
\tau^{\prime(1)}=\frac{z k^{3 / 2}}{\left(z^{2} k^{5 / 3}-z_{0}^{2} k_{0}^{5 / 3}\right)^{3 / 2}}\left(C_{\tau}^{(1)}-\frac{3}{2} \frac{z_{0} k_{0}^{5 / 6} \bar{q}_{0}^{2}}{k}\left(1-\frac{z^{2} k^{5 / 3}-z_{0}^{2} k_{0}^{5 / 3}}{k^{1 / 3} z^{2}}\right)\right)
$$


where $C_{\tau}^{(1)}$ is an integration constant. This expression seems to be divergent as $\mathcal{O}\left(\epsilon^{-3 / 2}\right)$ when $z$ is close to $z_{0}$ as $z=z_{0}+\epsilon$, and the degree of divergence is larger than that of $\tau^{\prime(0)}\left(z_{0}\right)$. Thus, we need to choose the integration constant as

$$
C_{\tau}^{(1)}=\frac{3}{2} z_{0} k_{0}^{-1 / 6} \bar{q}_{0}^{2}\left(z_{0}\right)
$$

Then the divergence becomes same as that of $\tau^{\prime(0)}\left(z_{0}\right)$. Since the value of $\tau^{\prime(1)}$ is always positive in any $z$ when $z_{0}=0$, we obtain

$$
\tau(\infty) \simeq \int_{0}^{\infty}\left(\tau^{\prime(0)}+\tau^{\prime(1)} n\right) d z>\frac{\pi}{2}
$$

Therefore, $\tau(\infty)$ is always larger than $\pi / 2$ at finite $n$. We can ensure the $n$-dependence

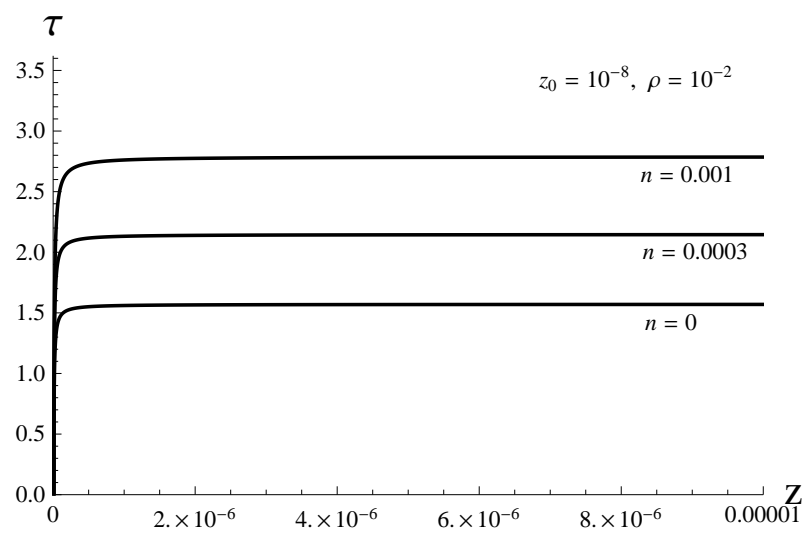

Fig. 6: Typical examples of the solutions of $\tau(z)$ for $z_{0}=10^{-8}$ and $\rho=10^{-2}$.

of $\tau(\infty)$ by numerical solutions. Typical examples are shown at small $z_{0}=10^{-8}$ for finite $n$ in Fig. 6. We assure that $\tau(\infty)$ increases with $n$.

\section{V-shaped antipodal solutions}

From the above result, the solution with $z_{0} \rightarrow 0$ at finite value of $n$ is not the antipodal solution. However, we can construct an antipodal solution by using this solution according to the idea of [8]. First, cut off the part of $z_{0}<z<z_{c}$ of the above solution for $D 8$, then connect it with the corresponding cut one of $\overline{D 8}$. This connected configuration has a $\mathrm{V}$ shape, and the point $z_{c}$ is chosen such that the the distance between two end points of $D 8$ and $\overline{D 8}$ becomes $\pi$, namely the antipodal configuration.

In general, however, the $\mathrm{V}$-shaped solution has a cusp at the connected point $z=$ $z_{c}\left(\geq z_{0}\right)$ because of $\tau^{\prime}\left(z_{c}\right) \neq \infty$, and they need a source term 6 in order to cancel this cusp singularity. Then, the total action should be replaced as

$$
S=S_{D B I}+S_{C S} \quad \Longrightarrow \quad \tilde{S}=S+S_{\text {source }} .
$$

\footnotetext{
${ }^{6}$ For example, we can consider the $D_{4}$ brane as in [8], however it is not necessary here to specify it.
} 
As we can see from the Fig. 6, the value of $z_{c}$ is estimated as $z_{c} \simeq z_{0}$. Then, the V-shaped solution is almost the same one with the simple solution given above. In addition, the $z$-component of the tension of the D8 brane on the cusp is negligible small because of $z^{\prime}(\tau) \simeq 0$ at the $z=z_{c}$, then the contribution of the source for the force balance conditions is negligible. Therefore, we can ignore the source term.

The next problem is to determine which antipodal solution we adopt in the present analysis. This is performed by comparing the energy densities of the two kinds of solution. As the example, we have compared the energy density $(\mathcal{E}(U, n))$ of the three examples given in the Fig. 4 with the $\mathrm{V}$-shaped $(\mathcal{E}(V, n))$ one given here, then we find $\mathcal{E}(U) / \mathcal{E}(V) \sim 20$. As shown in this example, the answer is that we should use the $\mathrm{V}$-shaped one with $z_{0} \rightarrow 0$ since its energy density is the lower than that of U-shaped one for finite $n$. And the adopted V-shaped solution is well approximated by our simple solution since the energy densities of them are almost equal as shown in the Fig. 7. Thus, the simple antipodal solution (3.22) is useful in our analyses for any value of $n$.

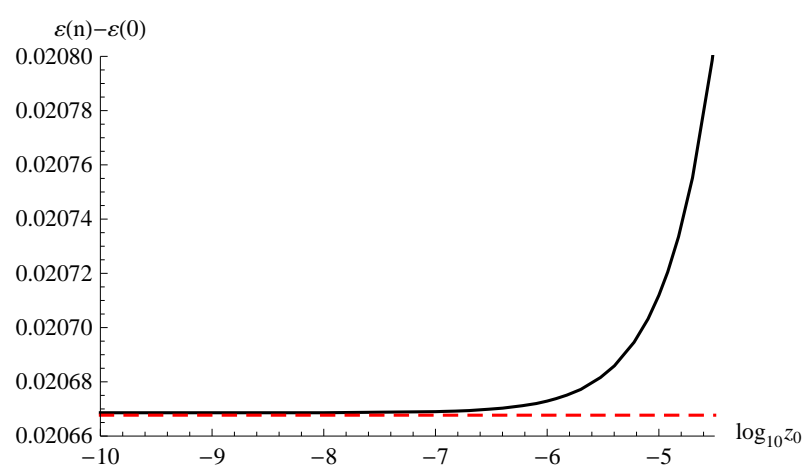

Fig. 7: V-shaped solution v.s. simple solution for $n=10^{-3}$ and $\rho=10^{-2}$. The curve denotes the $z_{0}$ dependence of $\mathcal{E}(n)-\mathcal{E}(0)$ of the $\mathrm{V}$-shaped one. The dotted (red) line denotes the value of $\mathcal{E}(n)-\mathcal{E}(0)$ of the simple solution.

\section{References}

[1] T. Schafer, "Phases of QCD," hep-ph/0509068.

[2] M. A. Stephanov, "QCD phase diagram: An Overview," PoS LAT 2006, 024 (2006) hep-lat/0701002.

[3] T. Sakai and S. Sugimoto, "Low energy hadron physics in holographic QCD," Prog. Theor. Phys. 113, 843 (2005) hep-th/0412141.

[4] H. Hata, T. Sakai, S. Sugimoto and S. Yamato, "Baryons from instantons in holographic QCD," Prog. Theor. Phys. 117, 1157 (2007) hep-th/0701280 [HEP$\mathrm{TH}]$ ]. 
[5] K. Hashimoto, T. Sakai and S. Sugimoto, "Holographic Baryons: Static Properties and Form Factors from Gauge/String Duality," Prog. Theor. Phys. 120, 1093 (2008) arXiv:0806.3122 [hep-th]].

[6] K. Hashimoto, T. Sakai and S. Sugimoto, "Nuclear Force from String Theory," Prog. Theor. Phys. 122, 427 (2009) [arXiv:0901.4449 [hep-th]].

[7] K. -Y. Kim, S. -J. Sin and I. Zahed, "Dense hadronic matter in holographic QCD," hep-th/0608046.

[8] O. Bergman, G. Lifschytz and M. Lippert, "Holographic Nuclear Physics," JHEP 0711, 056 (2007) [arXiv:0708.0326 [hep-th]].

[9] K. -Y. Kim, S. -J. Sin and I. Zahed, "Dense holographic QCD in the Wigner-Seitz approximation," JHEP 0809, 001 (2008) [arXiv:0712.1582 [hep-th]].

[10] M. Rozali, H. -H. Shieh, M. Van Raamsdonk and J. Wu, "Cold Nuclear Matter In Holographic QCD," JHEP 0801, 053 (2008) [arXiv:0708.1322 [hep-th]].

[11] W. -y. Chuang, S. -H. Dai, S. Kawamoto, F. -L. Lin and C. -P. Yeh, "Dynamical Instability of Holographic QCD at Finite Density," Phys. Rev. D 83, 106003 (2011) arXiv:1004.0162 [hep-th]].

[12] V. Kaplunovsky, D. Melnikov and J. Sonnenschein, "Baryonic Popcorn," JHEP 1211, 047 (2012) [arXiv:1201.1331 [hep-th]].

[13] S. Seki and S. -J. Sin, "Chiral Condensate in Holographic QCD with Baryon Density," JHEP 1208, 009 (2012) [arXiv:1206.5897 [hep-th]].

[14] J. de Boer, B. D. Chowdhury, M. P. Heller and J. Jankowski, "Towards a holographic realization of the Quarkyonic phase," arXiv:1209.5915 [hep-th].

[15] T. D. Cohen, "QCD functional integrals for systems with nonzero chemical potential," In *Shifman, M. (ed.) et al.: From fields to strings, vol. 1* 101-120 hep-ph/0405043.

[16] P. Haensel, A. Y. Potekhin and D. G. Yakovlev, "Neutron stars 1: Equation of state and structure," (Astrophysics and space science library. 326) 